ARTICULOORICINAL

\title{
Gobernanza territorial, oportunidades para el desarrollo y sostenibilidad en Santa Rosa de Copán, Honduras
}

\section{Territorial governance, opportunities for development and sustainability in Santa Rosa de Copán, Honduras}

Jensen Bautista-Perdomo ${ }^{\mathrm{a}, \mathrm{b}}$

a Carrera en Desarrollo Local, Centro Universitario Regional de Occidente, Universidad Nacional Autónoma de Honduras, Santa Rosa de Copán, Honduras

b Comisión Ciudadana de Desarrollo Urbano y Ordenamiento Territorial

DOI 10.5377/rct.v13i25.10413

Recibido: 23 octubre 2019

Aceptado: 03 de agosto 2020

Publicado en línea: 23 noviembre 2020

\section{RESUMEN}

En el presente artículo, se presenta un análisis sobre los planes de ordenamiento territorial como instrumentos actuales en la construcción de "un orden deseado" y sobre las posibilidades para lograr un modelo de ocupación que permita orientar el territorio hacia un desarrollo sostenible, con iniciativas de apropiación cultural, desarrollo social, desarrollo económico local y ambiental. Se sabe que aún existe un gran camino por recorrer hacia la implementación de una verdadera cultura de planificación y de gestión integral del territorio. En este contexto, el presente artículo presenta una propuesta de gobernanza territorial con un enfoque multidisciplinario, planteando 5 ejes de trabajo: cultura e identidad, planificación del crecimiento físico de la ciudad, transporte (viabilidad), gestión de riesgo y sostenibilidad ambiental (agua, saneamiento, ambiente y recolección de desechos sólidos), en donde se dan a conocer sus oportunidades. Esta propuesta de gobernanza territorial para el municipio de Santa Rosa de Copán, Honduras permitiría recuperar el equilibrio ambiental, la productividad económica, equidad social y los procesos de participación ciudadana.

Palabras clave: gobernanza, ordenamiento territorial, desarrollo sostenible, ambiente

\section{ABSTRACT}

The present article shows an analysis of territorial planningplansas current instruments in the construction of "a desired order" and on the possibilities to achieve an occupation model that allows guiding the territory towards a sustainable development, with initiatives of cultural appropriation, social development, local and environmental economic development. It is known that there is still a long way to go towards implementing a true culture of planning and integral management of the territory. In this context, this article presents a proposal of territorial governance with a multidisciplinary approach, proposing 5 axes of work: culture and identity, planning of the physical growth of the city, transport (viability), risk

Autor corresponsal

$\triangle$ Jensen Bautista-Perdomo

jensen.bautista@unah.edu.hn 
management, environmental sustainability (water, sanitation, environment and collection of solid waste), where the opportunities are revealed. This proposal of territorial governance for the municipality of Santa Rosa de Copán, Honduras would allow the recovery of the environmental balance, economic productivity, social equity and citizen participation processes.

Keywords: governance, land-use planning, sustainable development, environment

\section{INTRODUCCIÓN}

El término gobernanza, como menciona Dalla-Torre (2017), nos presenta una propuesta innovadora cuya finalidad es generar repuestas a la crisis existente en las formas tradicionales de gobierno. Esto permite formular las bases de nuevas instancias de legitimidad, participación y gobernabilidad, modificando nuevas relaciones entre el estado y la sociedad civil. UbillaBravo (2016) hace mención de los puntos necesarios para comprender qué es gobernanza: (1) es una estructura de relaciones entre los diferentes actores, (2) se encuentra formada por un conjunto articulado de reglas y procedimientos institucionalizados, (3) es compleja porque involucra a actores de los diferentes sectores: público, privado y social, (4) a menudo lo hacen con interdependencias multinivel, y (5) abarca a mecanismos relacionales mediante los cuales diversos actores intervienen en la toma de decisiones pública.

Existen muchos conceptos de desarrollo y sostenibilidad, pero en todas sus definiciones, se debe fundamentar en tres pilares, siendo estos las dimensiones ambientales, económicas y sociales. Estos tres pilares no pueden considerarse solos; más bien, es necesario tener en cuenta sus interacciones, permitiendo la creación, articulación y regulación de políticas y estrategias en un contexto de sistema complejo (Paolotti et al. 2019; Ferretti et al. 2020).
El concepto de desarrollo sostenible está estrechamente relacionado con el de sostenibilidad, es decir, la capacidad de cualquier sistema para mantenerse de forma indefinida. Esta definición ha evolucionado con el tiempo. La necesidad de preservar los recursos para las generaciones futuras es una de las principales características del desarrollo sostenible. Sin embargo, la única forma de lograr este objetivo es concebir el desarrollo como concepto multidimensional, teniendo en cuenta los tres pilares en los que se fundamenta. Para lograr el desarrollo sostenible, es necesario definir y delimitar metas y objetivos claros y que estos puedan ser medibles (Alaimo y Maggino 2019).

La estrategia de la gobernanza que surge por crisis ambientales, falta de gestión de recursos naturales y deterioro de los servicios ecosistémicos ha dado impulso a la territorialización de las políticas. Cada territorio, como actor global, puede escoger las trayectorias para el desarrollo, en las que la base identitaria y el apego al lugar guíen la acción y las políticas para poder alcanzar el futuro deseado. Esto debe ser respaldado por los múltiples actores que comparten los objetivos y conocen y asumen cuál debe ser su papel en su consecución (Farinós Dasí 2008). Esto nace ante la evidencia de las limitaciones de modelos de planificación y gestión pasados, los cuales han sido llevados a la práctica y han mostrado resultados, a menudo, alejados de los ambiciosos objetivos fijados. Se puede atribuir a la distancia y difícil coordinación entre las diferentes escalas administrativas (estatal, regional, local) y de estas con los agentes económico-sociales y población en general (Fernández Tabales et al. 2015).

En la planificación, se cuentan con áreas y sectores de las políticas públicas como la ambiental, social y territorial que, por su complejidad y la necesidad de legitimación permanente, son propicias para los modelos participativos o de gobernanza, 


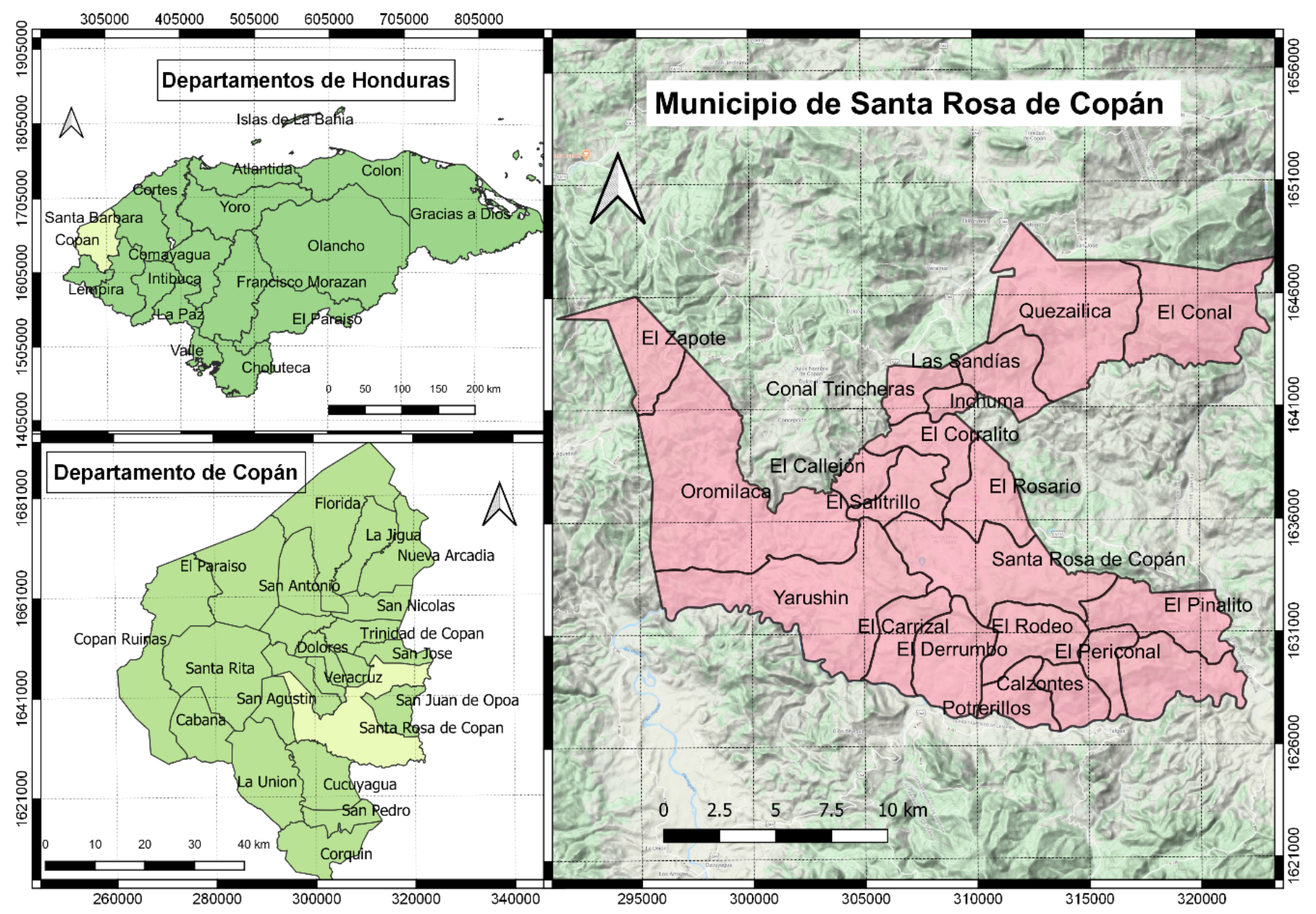

Fig. 1 Localización de Santa Rosa de Copán

la planificación y gestión del territorio. En estas se están generando instancias para las nuevas formas de gobierno local. Existe una problemática asociada a una incorrecta planificación urbana y del territorio y a la inapropiada interrelación estructural y funcional del uso de suelo urbano. Una indebida planificación ha llevado a una inapropiada expansión urbana y a la falta de planes de manejo territorial que sean integrales, trayendo consigo crisis ambientales por un deterioro de los bienes y servicios ecosistémicos. Esto conlleva situaciones en donde se crean condicionantes del desarrollo social y urbanístico como los procesos no planeados de transformación urbana, arrastrando espacios y ciudades mal planeadas (Ortega 2012; Farinós Dasí 2008; De Arco Ballesteros y Verga Durán 2012).

\section{2. ÁREA DE ESTUDIO}

Santa Rosa de Copán está situado en el extremo occidental de Honduras (ver Fig. 1). Es cabecera del Departamento de Copán y está ubicada en una altiplanicie extendida de oriente a poniente con una altura aproximada de 1,100 metros sobre el nivel del mar en las coordenadas $14^{\circ} 46^{\prime} 03^{\prime \prime}$ latitud norte y $88^{\circ} 46^{\prime} 41^{\prime \prime}$ longitud oeste. Presenta una topografía irregular, rodeada de bosques de coníferas. Su extensión 
superficial es de $293.1 \mathrm{~km}^{2}$. En el año 2017, según el Instituto Nacional de Estadística, poseía una población total (urbana y rural) de 66,629 habitantes.

\section{PLAN DE ORDENAMIENTO TERRITORIAL EN SANTA ROSA DE COPÁN}

De acuerdo al concepto de gobernanza, la sociedad civil debería jugar un papel muy importante en la toma de decisiones, con una inclusión participativa y de apoyo a las autoridades municipales. En Santa Rosa de Copán, existe la Agencia del Desarrollo Económico Local (ADELSAR), la cual es un instrumento de gestión con responsabilidad compartida entre ciudadanía, gobierno local y empresa privada. Dentro de esta, existen las Instancias Ciudadanas, que es la unidad encargada de articular el proceso de Participación Ciudadana del Municipio. A ella pertenece la Comisión Ciudadana de Desarrollo Urbano y Ordenamiento Territorial, que es un conjunto de ciudadanos voluntarios que buscan, como objetivo, asesorar el seguimiento y ejecución del Plan Maestro de Desarrollo Urbano Municipal (PMDU), el cual fue aprobado en el año 2010 con una proyección de 20 años. Esta comisión, al ser la encargada de velar por el cumplimiento y la actualización del PMDU, permite proponer y presentar a las autoridades locales, desde la ciudadanía, los cambios, modificaciones y revisión al mismo, buscando el desarrollo y la sostenibilidad. Esta comisión también se encarga de visualizar los retos y oportunidades para un desarrollo sostenible con un enfoque multidisciplinario para la ciudad de Santa Rosa de Copán, construyendo esta visión de territorio al PMDU actual de manera participativa.

Se reconoce que, a pesar de los avances en la materia, la planificación tradicional en general y el PMDU para la ciudad Santa Rosa de Copán, se encuentran ligados directamente al uso del suelo, sin contar con los componentes sociales, ambientales, culturales, etc. El proceso de planeación debe de ser multi e interdisciplinario. En el proceso, el intercambio de información y la comunicación clara y transparente entre todos los actores de Santa Rosa de Copán, tanto públicos como privados y civiles, son clave para generar la participación conjunta y la creación de una visión y los objetivos para el desarrollo sostenible. Es imprescindible sustentar y recopilar este proceso con datos técnicos y científicos e información clara sobre los servicios de los ecosistemas relevantes, espacios culturales y de recreación, el patrimonio histórico y los costes de gestión y sus beneficios sociales, ambientales y económicos. La sociedad civil, empresa privada y el gobierno local son la arquitectura de la gobernanza que podría estar compuesta por redes complejas de relaciones (horizontales y verticales) interinstitucionales o intersectoriales. Las relaciones, interacciones o transacciones (que también pueden darse) entre actores se pueden dar en varios temas o ejes: operacionales, financieros, legales, ambientales, etc. Estas relaciones se pueden llevar a cabo en diferentes niveles o dentro del mismo grupo de actores en diferentes tiempos.

En el PMDU de Santa Rosa de Copán, aprobado para el periodo 2010-2030, deberían cuestionarse algunas preguntas como:

- ¿Existe en Santa Rosa de Copán un modelo de participación y de gestión del territorio innovador y respaldado por un equipo multidisciplinario de actores?

- ¿El PMDU refleja una visión territorial compartida y consensuada por todo el municipio?

- ¿Es necesario modificar el plan maestro actual?

A modo de hipótesis, se sostiene que la gobernanza territorialen Santa RosadeCopán esun modeloemergente, aún limitado y de incipiente institucionalización. Aun no 
se ha logrado plasmar en el PMDU una visión clara a futuro sobre el territorio compartido y consensuada mediante la participación ciudadana, y siendo de manera incluyente en todos los ámbitos de la población.

Hablar de ordenamiento territorial o plan maestro de desarrollo urbano significa generar un proceso para la construcción del orden deseado. Esto solo se puede lograr con la participación de los grupos sociales y económicos, sus problemáticas, su razón de ser como ciudad o como grupo, con una identidad. No puede ser un ejercicio exclusivamente estadístico y normativo, sino que debe reconocer diferentes tendencias socioculturales, la historia y el modo de vida de la población (Hernández Peña 2010).

En este sentido, se realizó, a través del Centro Universitario Regional de Occidente (CUROCUNAH) y la Comisión Ciudadana de Desarrollo Urbano y Ordenamiento Territorial, una propuesta para adaptar el plan maestro actual para Santa Rosa de Copán. Se desea que el plan sea más integral, que englobe la realidad, que haga sentir la identidad y que sea complementario, buscando los procesos de planeación territorial, la historia, su patrimonio histórico y ambiental, los servicios ecosistémicos y socioculturales y la contribución a la discusión de alternativas.

El fenómeno urbano y su dinámica en cualquier parte del mundo se presenta como un proceso complejo de intercambio entre las personas, las actividades, el ambiente, los intereses, las culturas, los poderes, los deberes y derechos (Ornés 2009). Finalmente, el presente y futuro de Santa Rosa de Copán será una respuesta del compromiso colectivo, logrando una de las aportaciones relevantes de la nueva concepción de los procesos socio-ambientales. Es la idea de concebir al territorio como recurso y factor de desarrollo y no sólo como soporte físico para las actividades y los procesos económicos (Wong-González 2009).
La forma de planear para un presente y un futuro de la ciudad de Santa Rosa de Copán parte de posturas e intereses a veces encontrados. Es imperante realizar una reflexión $\mathrm{y}$, sobre todo, un diálogo sostenido entre los distintos actores sociales y políticos de Santa Rosa de Copán, sobre la planificación de la ciudad y la ciudad que se quiere. La ciudad de Santa Rosa de Copán presenta, alrededor del sistema de espacio público, un sinnúmero de actuaciones urbanísticas mal planificadas debido a su relieve irregular y a su acelerado crecimiento. Por ejemplo, las áreas de recreación, en su mayoría, se encuentran ubicadas en la zona alta de la ciudad. Los espacios correspondientes a cauces de agua están embaulados. El PMDU contempla actualmente solo el área urbana del municipio, obviando el área rural, lo que conlleva a un crecimiento sin una planificación establecida en las zonas rurales del municipio. Con gran indiferencia ante el actual sistema social para la convivencia, la materialidad urbana ha contribuido a la pérdida del sentido de pertenencia que debe primar en una ciudad con calidad ambiental, y donde la cultura debe ser la bandera de la convivencia pacífica y del hacer ciudad de todos.

El trabajo se inicia describiendo los problemas derivados de la falta de planificación urbana y ordenación del territorio en la ciudad de Santa Rosa de Copán, y que repercuten en la progresiva pérdida de calidad de vida de los ciudadanos. Asimismo, se enfatiza sobre los desafíos, retos y oportunidades a los que se enfrentan los ciudadanos y el gobierno local, reivindicando la reflexión y la búsqueda que dé respuestas a las necesidades locales y brinde desarrollo a las poblaciones heterogéneas que comparten el espacio urbano.

La planificación del desarrollo urbano en Santa Rosa de Copán se justifica porque:

a. son pocos los espacios habitables en relación con la superficie de la ciudad, ya que en 
Santa Rosa de Copán existen muchas áreas escarpadas y erosionables

b. existen zonas cuyas características demandan tratamientos especiales, por ejemplo, las zonas de recarga, áreas de recreación, áreas boscosas, etc.

Las decisiones que se tomen definirán el futuro de manera determinante para la ciudad de Santa Rosa de Copán.

\section{LOS DESAFÍOS DE LA PLANIFICACIÓN URBANA EN SANTA ROSA DE COPÁN}

El gran desafío de la planificación urbana en Santa Rosa de Copán es garantizar ser consecuente con las necesidades de la sociedad en su conjunto, respetuosa con la legislación urbana, coherente en las técnicas y metodologías empleadas en el proceso de planificación, seguimiento y monitoreo, y, sobre todo, independiente de los que ejecutan y desarrollan los planes. En este sentido, en el proceso de planificar aparecen intereses en pugna, por lo que es fundamental yuxtaponer los deseos opuestos de la administración local por planear a corto plazo y los anhelos de los distintos espacios urbanos por ser diseñados a largo plazo (Sánchez González 2012).

El crecimiento urbano ejerce presión sobre los recursos y la capacidad de los planificadores y las autoridades locales para mejorar las condiciones de los ciudadanos. El desarrollo sostenible depende, por tanto, de la gestión exitosa de la expansión urbana y el desarrollo de estrategias eficientes de planificación territorial (Billaud et al. 2020).

Algunos investigadores (Hernández Peña 2010; Sánchez González 2012) indican que el aumento indiscriminado de la población urbana ha traído consigo la pérdida progresiva de la calidad de vida urbana y regional. Asimismo, la ciudad actual se ha convertido en el escenario crítico de una sociedad inconforme y consumista de recursos finitos (suelo, agua). El conflicto se da entre las posibilidades de conservar los recursos y condiciones ambientales, y continuar con un modelo de producción económico cuyos costos de producción no contemplan los costos ambientales de estas actividades. Dentro de este desafío de crecimiento de ciudad, es necesario visualizar las metas de los Objetivos de Desarrollo Sostenible (ODS 11), buscando una urbanización inclusiva y sostenible. Se debe considerar, además, la capacidad para una planificación y gestión participativa, integrada y sostenible, basada en el apoyo a los vínculos económicos, sociales y ambientales positivos entre las zonas urbanas, periurbanas y rurales mediante el fortalecimiento de la planificación desde una visión de desarrollo nacional y regional (ONU 2015). La Nueva Agenda Urbana permite definir lineamientos para el desarrollo sostenible, en la forma de administrar, planificar y gestionar las ciudades, incorporando un enfoque de gestión integral, hacia ciudades más sostenibles y armonía permitiendo el uso sostenible de sus recursos (ONU-HABITAT 2016). Esto conduce a un proceso de planificación a nivel de territorio, permitiendo construir en consenso y con una visión compartida en el municipio de Santa Rosa de Copán.

\section{OPORTUNIDADES EN EJES DE TRABAJO Y DESARROLLO}

Existe una creciente necesidad de habilidades prácticas y grupos de expertos calificados para desarrollar, implementar y operar modelos y soluciones de gobernanza para propósitos colectivos específicos y en contextos geográficos particulares. La gobernanza significa más que gobernar y gestionar, ya que implica la capacidad de coordinación de las diferentes organizaciones y sus intereses particulares dentro de un marco institucional específico (Glückler 
et al. 2019).

Por tal razón, para que Santa Rosa de Copán logre transitar a procesos de innovación inclusiva, se requiere necesariamente pensar en la conformación de líneas de actuación (abordado también como ejes de trabajo). Esto se refiere a la articulación/ integración entre los diferentes actores y estructuras institucionales con la cual se busca explicar su interacción dentro del proceso de innovación. Este es un proceso social e interactivo en un espacio social específico. En este aspecto, la comisión ciudadana de desarrollo urbano y ordenamiento territorial propone un cambio al PMDU para crear un plan de desarrollo que involucre a la sociedad, la cultura, el ambiente y el desarrollo sostenible. Con esto, se busca dar solución a los problemas existentes, construyéndose a través de la participación ciudadana. Dentro de estos ejes se encuentran:

\subsection{Eje de trabajo 1: Cultura e Identidad}

Este primer eje de trabajo deberá hacer énfasis en el ámbito cultural y de identidad en Santa Rosa de Copán. La cultura se ha convertido en uno de los elementos decisivos en el desarrollo económico, social y de la identidad. En este sentido, las políticas culturales deberían efectuar un giro local hacia el café, el cual ha sido fuente de emprendedurismo. Cultura e identidad son dos términos que, en el caso de Santa Rosa de Copán, debería de ser indisociables. La primera función de la identidad es marcar fronteras entre un nosotros y los "otros" y no se ve de qué otra manera podríamos diferenciarnos de los demás si no es a través de un universo de rasgos culturales distintivos y muy marcados de la ciudad.

El desarrollo sostenible para Santa Rosa de Copán es concebido como la integración equilibrada entre un desarrollo económico de cultura e identidad que permita obtener calidad de vida para la población local y aquel en el que, al mismo tiempo, se preserve el ambiente. Estos aspectos en conjunto son la base del desarrollo sostenible que, bajo una adecuada planificación, se puede llegar a conseguir. El turismo, visto desde este punto, es una de las industrias más lucrativas debido a su gran acogida, y donde Santa Rosa de Copán puede ver una oportunidad de desarrollo en sus recursos naturales y culturales. La Comisión de Turismo, la Cámara Nacional de Turismo de Honduras (CANATURH-SRC), la Asociación de Cafeterías de Occidente y el Club de Observación de Aves (COAS) están trabajando fuertemente en el turismo sostenible y con una visión integradora donde lo social, el ambiente y la cultura están de la mano.

Se deberá hacer un énfasis en el patrimonio, ya que a nivel territorial, se refiere a aquellos bienes que una sociedad decide conservar por su valor cultural, natural o tradicional. Se plantea que el patrimonio urbano "comprende las edificaciones y los espacios públicos cuya forma constitutiva es expresión de la memoria colectiva, arraigada y trasmitida, los que en forma individual o en conjunto revelan características culturales, ambientales y sociales que manifiestan y fomentan la cultura y la consolidación social" (Camelo Garzón et al. 2015).

Dentro de las oportunidades para el Eje 1 se destacan:

a. Crear una ciudad sostenible

- Implementar estrategias para el desarrollo sostenible con un crecimiento constante y en armonía con el ambiente

- Considerar una estructura urbana que contribuya al desarrollo del futuro

b. Crear una ciudad económicamente activa y atractiva

- Proponer un concepto de identidad para la ciudad (por ejemplo, las actividades que la sociedad civil está realizando: "la capital del buen café”, Expocopán y la fabricación 
de puros)

- Ampararse en los lineamientos del patrimonio histórico

- Cuidar los recursos naturales y medioambiente natural

c. Implementar un plan de recuperación de zona (casco histórico) y patrimonio arquitectónico

d. Crear una ciudad turística, con base en las debilidades y fortalezas

- Realizar un análisis del sector turismo

- Definir las oportunidades y retos, entre ellos:

- Recursos turísticos

- Oferta turística

- Infraestructura de apoyo

- Problemática del centro de Santa Rosa de Copán (casco histórico)

- Proyectos-destinos estratégicos (por ejemplo, rutas de café)

- Plan de marketing (por ejemplo, mercados, estrategias, acciones).

\subsection{Eje de trabajo 2: Planificación del crecimiento físico de la ciudad}

Las ciudades actuales son fruto de combinaciones de fenómenos urbanos pasados y nuevos. Santa Rosa de Copán se encuentra en una dinámica continua y en un acelerado movimiento. Algunos han creado un paradigma en cuanto a que dichas dinámicas necesariamente se deben orientar hacia un determinado tipo de desarrollo, a veces entendido como "crecimiento económico".

El crecimiento físico de la ciudad debe estar acondicionado para brindar servicios a la ciudad y mejorar la calidad de vida de la población, pero se necesita una clara visión de las necesidades de la ciudad y hacia dónde avanza. Esto implica dimensiones materiales, ambientales y espaciales (equipamientos). Para este eje de trabajo, se necesita establecer la calidad de vida de la población de Santa Rosa de Copán, medida desde la satisfacción de necesidades materiales, a través de indicadores externos a la persona (por ejemplo, calidad de vivienda, acceso a servicios públicos, acceso a zonas de ocio, ingresos, etc.). Esto le brinda un lugar protagónico a los espacios públicos destinados a satisfacer las necesidades fundamentales que producen bienestar de la población. También se debe incluir otro tipo de mediciones o indicadores que evalúen el grado de felicidad de la población de Santa Rosa de Copán (por ejemplo, su tiempo libre, sus oportunidades de recreación, acceso a la cultura y otros).

El espacio, cada vez más urbanizado, hace necesario realizar importantes procesos de adaptación, aunque también es susceptible de ser transformado para hacerse más sostenible. Una de las preocupaciones que surge al pensar en la crisis ambiental y de planificación actual es que se ha ido perdiendo control sobre el entorno en el cual se vive. Por esta razón, Santa Rosa de Copán necesita tener una clara visión hacia dónde va a avanzar y cuáles son los pasos a seguir. Es necesario contar con un estudio que contemple el uso del suelo y la estructuración en el espacio para definir las características de sus elementos (calles, plazas, parques, edificación, áreas verdes). De esta manera, se debe tratar de poner racionalidad al conjunto urbano, mejorando las condiciones de vida y llegando a satisfacer las necesidades básicas dentro del espacio público.

ElEje 2 aborda fuertemente el tema de equipamiento público en la planificación y ordenamiento de los 
territorios, ya que permite establecer la infraestructura necesaria para que su población cuente con los servicios básicos de salud, educación, cultura, recreación y bienestar social, entre otros. Se deben establecer estándares mínimos y lugares adecuados para que los ciudadanos tengan acceso a estos servicios indispensables para su calidad de vida y su bienestar general.

Dentro de las oportunidades para el Eje 2 se destacan:

a. Indicar necesidades claras

- Identificar funciones urbanas necesarias para la ciudad de Santa Rosa de Copán.

- Considerar medidas necesarias para llevar a cabo dichas funciones

- Elaborar un plan sectorial de los espacios públicos, vinculando el desarrollo urbano ecológico, de áreas verdes y recreación

- Establecer mecanismos financieros sostenibles y transparentes

b. Introducir un método participativo efectivo de los actores principales

- Involucrar a los interesados en la formulación de los planes de uso de suelo

- Llevar a cabo un proceso participativo de un consenso que involucre a diversos actores

- Actualizar el Reglamento de Construcción y Lotificación con nuevas propuestas

- Fortalecer las instituciones relacionadas con la planificación y el desarrollo

c. Realizar propuesta de zonificación del uso de suelo

- Revisar antecedentes
- Desarrollar una base de datos en un sistema de información geográfica (SIG)

- Crear zonas para crecimiento poblacional

- Ubicar y conocer las limitaciones geomorfológicas

- Compartir y utilizar los datos en SIG entre las instituciones

- Proponer mecanismos de compensación en las áreas de desarrollo forestal con potencial para proyectos de ecoturismo, etc.

- Identificar áreas verdes y de recreación (por ejemplo, El Cerrito y el Bosque Escuela Jerónimo J. Reina)

d. Orientar el crecimiento de la ciudad hacia la creación de áreas residenciales eficientes que permitan un fácil acceso a las zonas de empleo, comercio, equipamiento y recreación.

\subsection{Eje de trabajo 3: Transporte (viabilidad)}

Estudiar este tema desde el desarrollo urbano permite evidenciar el papel estratégico de los proyectos de infraestructura para viabilidad, así como su capacidad para estructurar el territorio y establecer un primer nivel de ordenamiento territorial. El tema de transporte en el ordenamiento territorial tiene que ver con la eficiencia y eficacia de las infraestructuras y/o sistemas viales y de transporte que inciden directamente en la productividad de Santa Rosa de Copán y en las condiciones de vida de sus pobladores. Se analizan bajo esta temática principalmente los sistemas de infraestructuras viales y de movilidad, incluyendo la movilidad de peatones, automóviles, sistemas de transporte masivo, bicicletas, y las diferentes alternativas de transporte.

Dentro de las oportunidades para el Eje 3 se 
destacan:

a. Realizar un estudio de tráfico y pronóstico de tráfico

b. Revisar el sistema de transporte actual

c. Realizar propuesta de infraestructura física y no física (infraestructura de carreteras, bulevares, etc)

d. Introducir parquímetros

e. Implementar transporte limpio

f. Promover seguridad vial.

\subsection{Eje de trabajo 4: Gestión de riesgo}

El riesgo de desastre es una condición emergente del desarrollo y, por lo tanto, la gestión del riesgo es un proceso social y una estrategia de desarrollo que se concreta en diferentes niveles de integración ambiental, económica, política y sociocultural (Ramírez et al. 2005). Dentro de la gestión de riesgo, no solamente se estudian los fenómenos biofísicos; se deben abordar desde una visión más integral y sistemática, reconociendo las relaciones entre diversas variables sociales, económicas, ambientales y biofísicas, considerando múltiples escalas de tiempo y espacio. Esta visión, producto de una construcción social con diferentes visiones del fenómeno, crea una visión que permite analizar las características del riesgo, anteriormente ignoradas, y que están relacionadas con el carácter de complejidad e incertidumbre, generando una percepción más amplia del riesgo con nuevas estrategias para gestionarlo.

El riesgo en Santa Rosa de Copán deberá ser analizado como un producto de procesos complejos en la parte social. La identificación de la vulnerabilidad social empieza a ser clave en el análisis del riesgo, donde la pobreza, la inseguridad, la desigualdad social, el desempleo, el analfabetismo, la ausencia de infraestructura, la corrupción y la estructura de gobierno son variables sociales que claramente deben ser tomadas en cuenta con el objetivo de generar resiliencia y adaptación territorial. Para la ciudad de Santa Rosa de Copán, la sociedad civil necesita crear estrategias de empoderamiento en los sistemas de alerta, la detección y reconocimiento de amenazas, la actuación durante las emergencias y la educación ambiental. Esto permitirá que exista una redistribución de responsabilidades entre los múltiples actores a nivel municipal e instituciones de gobierno, creando vínculos de cooperación entre ellos.

Dentro de las oportunidades para el Eje 4 se destacan:

a. Generar conocimiento científico y sobre reducción del riesgo, generando estrategias y mecanismo para anticiparse a los desastres, a través de políticas públicas de gestión y reducción de riesgo

b. Crear la Comisión Ciudadana de Riesgos, la cual debería ser un grupo multidisciplinario en la que participen los diferentes actores y organizaciones sociales relacionadas con la gestión del riesgo, identificando posibles aliados, conflictos, intereses comunes, interdependencias y potenciales interacciones, tratando de establecer conexiones entre la comunidad científica, el sector público y el sector privado

c. Generar mapas de riesgo enfocados a determinar la probabilidad de las amenazas y el nivel de impacto que tendrían sobre el municipio de Santa Rosa de Copán ante la vulnerabilidad y el grado de exposición de la sociedad

d. Generar mapas de vulnerabilidad social enfocados a identificar las zonas vulnerables 
dentro del municipio de Santa Rosa de Copán

e. Crear un plan de acción que permita enfocar la gestión del riesgo bajo el conocimiento del riesgo, reducción del riesgo y manejo de la emergencia, con la finalidad de producir participación y consenso de la sociedad civil en la toma de decisiones.

\subsection{Eje de trabajo 5: Sostenibilidad Ambiental (Agua, Saneamiento, Ambiente y Recolección de desechos sólidos)}

Muchos de los problemas de Santa Rosa de Copán se derivan del propio emplazamiento y condiciones topográficas del sitio. La vulnerabilidad ambiental está dada no sólo por las características topográficas mencionadas, sino también por las relaciones sociales, culturales, económicas y políticas que nos conducen a la forma y modalidad de ocupación y crecimiento urbano que tenemos actualmente.

Dentro del eje 5, se trabajará con los componentes:
a. Agua
b. Saneamiento
c. Ambiente
d. Recolección de desechos sólidos.

\subsubsection{Agua}

El proceso de asentamiento humano de Santa Rosa de Copán tiene características muy particulares. Las condiciones de vulnerabilidad hídrica del sitio en el que se emplaza están dadas por las características topográficas y la disposición de los asentamientos urbanos, ocupando la planicie en un principio. El centro histórico de la ciudad está asentado sobre material volcánico donde existen acuíferos fracturados. Sus quebradas están contaminadas por aguas residuales y sin fuentes cercanas de aguas superficiales. Todo esto contribuye a la situación de riesgo hídrico que actualmente existe, como menciona Bautista-Perdomo y Martínez-Villegas (2015). Dentro de las oportunidades para abordar este componente se encuentran:

- Cobertura

- Eficiencia en el uso del agua

- Eficiencia en el servicio de suministro del agua

- Disponibilidad del recurso hídrico

- Delimitación de zonas no negociables

- Déficit en el servicio de agua potable de un 44 $\%$ (2014)

- Conservación, protección, y restauración (zonas de recarga) y aprovechamiento sostenible del agua

- Desarrollo de infraestructura para el tratamiento, uso y reúso eficiente de agua pluviales, subterráneas y residuales

- Abastecimiento y presión de agua disponibles para la etapa de desarrollo

- Creación de mecanismos de compensación por servicios ecosistémicos.

\subsubsection{Saneamiento}

La Cooperación Española se encuentra trabajando en el Proyecto Construcción del Plan Maestro (director) del Alcantarillado Sanitario, con la instalación de colectores en las dos quebradas que atraviesan la ciudad, las cuales recogerán las aguas negras de las colonias y barrios que descargan directamente a las quebradas. Esto vendrá a mejorar la calidad de vida para la sociedad, el ambiente, el recurso hídrico y el saneamiento. Posteriormente, se tiene planificada la 
construcción de una planta de tratamiento de aguas residuales (PTAR), como parte de un proceso de descontaminación del agua residual.

Dentro de las oportunidades se encuentran:

- Cobertura de saneamiento

- Tratamiento de aguas residuales

- Efectividad del drenaje

- Mantenimiento a la planta de tratamiento de aguas residuales.

\subsubsection{Ambiente}

Ante la perspectiva de crecimiento futuro, y considerando la necesidad de salvaguardar la parte ambiental a la par de planear el desarrollo para Santa Rosa de Copán, el ordenamiento ambiental del territorio deberá incluir la zonificación y la propuesta de usos para el territorio. Esto debe realizarse de acuerdo a su potencialidad y limitantes, así como el sistema de políticas ambientales de aprovechamiento, protección, conservación y restauración que garanticen la explotación racional y la conservación de los recursos naturales a mediano y largo plazo.

En el ordenamiento territorial, se considera el suelo como un recurso natural y se debe planear $\mathrm{y}$ ordenar todos sus usos en forma integral. Esta integración debe hacerse atendiendo, de un lado, todos los factores ambientales, sociales y económicos y, por otro, todos los componentes del medio ambiente y los recursos conjuntamente.

Dentro de las oportunidades se encuentran:

- Desarrollar incentivos fiscales o de otra índole que apoyen acciones de protección a los ecosistemas y su función en el ciclo hidrológico

- Garantizar el respeto a las áreas naturales protegidas

- Promover la compra de zonas de protección ambiental, mediante fondos municipales $y$ privados, con el fin de conformar cinturones verdes y zonas de amortiguamiento ecológico

- Generar y gestionar información para la toma de decisiones

- Desarrollar programas de reforestación en áreas degradadas en las zonas de captación hidrológica para incrementar la cantidad de áreas verdes y la capacidad de absorción de los suelos

- Establecer convenios con instituciones para que concentre, genere, actualice y difunda la información medioambiental.

\subsubsection{Recolección de desechos sólidos}

Una de las preocupaciones que surge al pensar en la crisis ambiental actual es que el hombre ha ido perdiendo control sobre el entorno en el que vive. Los residuos han ido incrementándose a medida crece la población de Santa Rosa de Copán.

Dentro de las oportunidades se encuentran:

- Crear programas de separación selectiva de basura y reciclaje

- Mejorar la cobertura de recolección de residuos sólidos.

- Implementar el tratamiento de residuos sólidos.

La propuesta de ordenamiento territorial para el eje 5 deberá estar compuesta por:

a. Unidad ambiental de gestión territorial

b. Política ambiental 
c. Escenarios actuales, tendencial y estratégico

d. Modelo de desarrollo sostenible.

\section{CONCLUSIONES}

La propuesta pretende realizar una evaluación integral a la gestión del desarrollo urbano, organizando los aspectos culturales, ambientales, sociales, económicos y científicos, los cuales abarcan todos los procesos que implica el desarrollo físico de los territorios. Así mismo, de manera transversal, la metodología propuesta evalúa, en cada uno de estos niveles, las grandes temáticas o dimensiones del ordenamiento, las cuales pueden ser evaluadas por separado y de este modo encontrar problemáticas puntuales y definir áreas a priorizar.

La sostenibilidad para Santa Rosa de Copán deberá ser una unión compleja entre la parte ambiental, la parte económica y la parte social. En este marco dinámico, flexible, descentralizado y participativo, se debe vincular en forma coherente y comprehensiva el proceso de desarrollo regional-territorial con el ordenamiento de la ciudad, particularmente bajo un esquema nacional de política regional y dentro de un contexto de globalización.

Ante todo, debe concebirse el ordenamiento como un instrumento que permite, a través de un ejercicio prospectivo, generar un modelo de planificación territorial donde se fortalecen las vocaciones de los territorios, y es posible tener criterios técnicos para subsanar los conflictos en el uso de los suelos, armonizando y salvando zonas de interés ambiental y cultural. En este sentido, el ordenamiento no debe concebirse como una obligación más, sino como un instrumento que permita construir sobre un territorio un orden deseado y orientado hacia el horizonte de una sustentabilidad ambiental.

La aplicación de políticas públicas de gestión del riesgo debe facilitar y demandar la participación corresponsable de los actores sociales, a efectos de concertar intereses y pactar compromisos para la mutua gestión. Se necesita crear mecanismos de supervisión y seguimiento para cumplir con los objetivos de cada propuesta.

Se hace necesario realizar importantes procesos de adaptación en el espacio de Santa Rosa de Copán, el cual está cada vez más urbanizado, aunque también es susceptible de ser transformado para hacerlo más sostenible. Se trata de una realidad que estimula a repensar las relaciones entre sociedad y espacio.

La municipalidad y los entes de gobierno no pueden entregar por sí solos una respuesta adecuada a las problemáticas antes descritas. Surge la necesidad de planificar con un enfoque ampliamente participativo.

\section{REFERENCIAS BIBLIOGRÁFICAS}

Alaimo, L. S., \& Maggino, F. (2019). Sustainable development goals indicators at territorial level: Conceptual and methodological issues-the Italian Perspective. Social Indicators Research, 147(2), 383-419. doi: 10.1007/ s11205-019-02162-4

Bautista-Perdomo, J. M., \& Martínez-Villegas, N. (2015). Santa Rosa de Copán; una propuesta hacia una nueva gestión del agua. Economía y Administración (E\&A), 6(1), 53-62

Billaud, O., Soubeyrand, M., Luque, S., \& Lenormand, M. (2020). Comprehensive decision-strategy space exploration for efficient territorial planning strategies. Computers, Environment and Urban Systems, 83, 101516. doi: 10.1016/j. compenvurbsys.2020.101516

Camelo Garzón, Á., Solarte-Pazos, L., \& López, O. (2015). Evaluación y seguimiento de planes de ordenamiento territorial en los municipios de Colombia. Revista Sociedad y Economía, (28), 163-180

Dalla-Torre, M. A. (2017). Gobernanza territorial y los Planes de Ordenamiento Territorial: el caso de la provincia de Mendoza, Argentina. Bitácora Urbano Territorial, 27(1), 47-54. doi: 10.15446/bitacora.v27n1.47597

De Arco Ballesteros, J. L. \& Verga Durán, R. A. (2012). Ordenamiento territorial, desarrollo social y construcción 
de patrimonio en el Suroccidente de Barranquilla. Caso: Barrio Los Ángeles. Memorias. Revista Digital de Historia y Arqueología desde el Caribe, (18), 168-195

Farinós Dasí, J. (2008). Gobernanza territorial para el desarrollo sostenible: estado de la cuestión y agenda. Boletín de la A. G. E. 46: 11-32. Recuperado de: http://age.ieg.csic.es/ boletin/46/02-GOBERNANZA.pdf

Fernández Tabales, A., Mercado Alonso, I., Villar Lama, A. \& Bascarán Estévez, M. V. (2015). Gobernanza territorial y gestión de espacios turísticos en contextos de fuerte presión inmobiliaria: Análisis de buenas prácticas locales en la costa de Cádiz (España). Revista de Geografía Norte Grande, (60), 173-194. doi: 10.4067/S0718-34022015000100010

Glückler, J., Rehner, J., \& Handke, M. (2019). Gobernanza, redes y territorio. Revista de Geografia Norte Grande, (74), 5-20. doi: 10.4067/S0718-34022019000300005

Hernández Peña, Y. (2010). El ordenamiento territorial y su construcción social en Colombia: ¿un instrumento para el desarrollo sustentable? Cuadernos de Geografia: Revista Colombiana de Geografia, (19), 97-109. doi:10.15446/ $\underline{\text { rcdg.n19.16854 }}$

ONU (2015). Objetivos de Desarrollo Sostenible. Consultado en: http://www.un.org/sustainabledevelopment/es/objetivosde-desarrollo-sostenible/

ONU-HABITAT (2016). Habitat III. Nueva Agenda Urbana. Nairobi: Organización de las Naciones Unidas. Consultado en: http://habitat3.org/wp-content/uploads/NUA-Spanish. pdf

Ornés, S. (2009). El urbanismo, la planificación urbana y el ordenamiento territorial desde la perspectiva del derecho urbanístico venezolano. Politeia, 32(42), 197-225

Ortega, A. C. (2012). Desarrollo territorial rural y estructuras de gobernanza en Brasil. Economía, sociedad y territorio, 12(38), 149-179

Ferretti, P., Zolin, M. B., \& Ferraro, G. (2020). Relationships among sustainability dimensions: evidence from an Alpine area case study using Dominance-based Rough Set Approach. Land Use Policy, 92, 104457. doi: 10.1016/j. landusepol.2019.104457

Paolotti, L., Del Campo Gomis, F.J., Agullo Torres, A.M., Massei, G., \& Boggia, A., (2019). Territorial sustainability

80 evaluation for policy management: the case study of Italy and Spain. Environmental Science \& Policy, 92, 207-219. doi: 10.1016/j.envsci.2018.11.022

Ramírez, F., Ghesquiere, F. \& Costa, C. (2005). Un modelo para la planificación de la gestión del riesgo de desastre en grandes ciudades. Recuperado de http://www.ceped. ufsc.br/wp-content/uploads/2014/07/un_modelo_para la planificacion_de la la gestion_del_riesgo_de_desastre_em grandes ciudades.pdf

Sánchez González, D. (2012). Aproximaciones a los conflictos sociales y propuestas sostenibles de urbanismo y ordenación del territorio en México. Revista de Estudios Sociales, (42), 40-56. doi: 10.7440/res42.2012.05

Ubilla-Bravo, G. (2016). Gobernanza territorial: bases, características y la necesidad de su estudio en Chile. Seminario Sustentabilidad y Gobernanza, Universidad de Chile, Sep 2016, Santiago, Chile. doi: 10.5281/zenodo.46703

Wong-González, P. (2009). Ordenamiento ecológico y ordenamiento territorial: retos para la gestión del desarrollo regional sustentable en el siglo XXI. Estudios sociales (Hermosillo, Son.), 17(spe), 11-39 